\title{
The anti-apoptotic, antioxidant and anti- inflammatory effects of curcumin on acrylamide-induced neurotoxicity in rats
}

\author{
Jie Guo ${ }^{1,2}$, Xiaolu Cao ${ }^{1,2}$, Xianmin $\mathrm{Hu}^{1,2}$, Shulan $\mathrm{Li}^{1,2}$ and Jun Wang ${ }^{1,2^{*}}$
}

\begin{abstract}
Background: Acrylamide (ACR) formed during heating of tobacco and carbohydrate-rich food as well as widely applied in industries has been known as a well-established neurotoxic pollutant. Although the precise mechanism is unclear, enhanced apoptosis, oxidative stress and inflammation have been demonstrated to contribute to the ACRinduced neurotoxicity. In this study, we assessed the possible anti-apoptotic, antioxidant and anti-inflammatory effects of curcumin, the most active component in a popular spice known as turmeric, on the neurotoxicity caused by ACR in rats.
\end{abstract}

Methods: Curcumin at the dose of 50 and $100 \mathrm{mg} / \mathrm{kg}$ was orally given to ACR- intoxicated Sprague-Dawley rats exposed by ACR at $40 \mathrm{mg} / \mathrm{kg}$ for 4 weeks. All rats were subjected to behavioral analysis. The HE staining and terminal deoxynucleotidyl transferase mediated dUTP nick end labelling (TUNEL) staining were used to detect histopathological changes and apoptotic cells, respectively. The mRNA and protein expressions of apoptosis-related molecule telomerase reverse transcriptase (TERT) were detected using real-time PCR and immunohistochemistry, respectively. The contents of malondialdehyde (MDA) and glutathione (GSH) as well as the activities of superoxide dismutase (SOD) and glutathione peroxidase (GSH-Px) were measured as the indicators for evaluating the level of oxidative stress in brain. The levels of pro-inflammatory cytokinestumor necrosis factor-a (TNF- $\alpha$ ) and interleukin-1 $\beta$ (IL-1ß) in the cerebral homogenates were detected using ELISA assay.

Results: ACR-induced weigh loss, deficits in motor function as well as pathological alterations in brains were significantly improved in rats administrated with 50 and $100 \mathrm{mg} / \mathrm{kg}$ curcumin. TUNEL-positive apoptotic cells in curcumin-treated ACR intoxicated brains were less than those in the ACR model group. Curcumin administration especially at the dose of $100 \mathrm{mg} / \mathrm{kg}$ upregulated the TERT mRNA expression and enhanced the number of TERTpositive cells in ACR-intoxicated cortex tissues. Moreover, curcumin treatment reduced the concentrations of TNF-a, $I L-1 \beta$ and MDA, while increased the GSH contents as well as the SOD and GSH-Px activities in the cerebral homogenates, in comparison to ACR control group.

(Continued on next page)

\footnotetext{
* Correspondence: wangjun@wust.edu.cn

'Hubei Province Key Laboratory of Occupational Hazard Identification and Control, Wuhan University of Science and Technology, Wuhan 430065, China ${ }^{2}$ Department of Pharmacy, New Medicine Innovation and Development Institute, College of Medicine, Wuhan University of Science and Technology, Wuhan 430065, China
} 
(Continued from previous page)

Conclusions: These data suggested the anti-apoptotic, antioxidant and anti-inflammatory effects of curcumin on

ACR-induced neurotoxicity in rats. Maintaining TERT-related anti-apoptotic function might be one mechanism underlying the protective effect of curcumin on ACR-intoxicated brains.

Keywords: Acrylamide, Curcumin, Apoptosis, Antioxidant, Inflammation, Telomerase reverse transcriptase

\section{Background}

As a chemical formed during the high-temperature processing of tobacco and carbohydrate-rich foods, acrylamide (ACR) is well recognized as a human neurotoxin which has posed significant public health concerns due to its daily intake [1-3]. Moreover, ACR is widely employed in various chemical and industrial processes as a component to produce polymers used in gel chromatography, dye synthesis, production of paper, cosmetics and waste water management, etc [4, 5]. The work-related ACR exposure has been demonstrated to bring on neurotoxicity in occupationally exposed population, which is manifested as ataxia, skeletal muscle weakness, gait abnormalities, skin abnormalities, as well as numbness of hands and feet [4].

The exposure to monomeric form of ACR results in multiple pathological changes in central and peripheral nervous system. Among them, ACR-induced apoptosis that subsequently leads to the death and loss of neurons has been accepted as a fundamental and predominant mechanism of neurotoxicity in ACR-exposed humans and animals [6-8]. Telomerase reverse transcriptase (TERT) is one of catalytic units of telomerase, importantly, acts as rate-limiting determinant and the most important regulator of telomerase activity $[9,10]$. Telomerase is required to synthesize the telomeric DNA strand thus maintain the length of telomeres, the latter of which is a DNA-protein complex located at chromosome ends and has an ability of protecting against genome instability [9]. So far, the anti-apoptotic effect of TERT has been revealed in neuronal cells influenced by various risk factors such as oxidative stress, DNA damage and ischemia $[9,10]$. In line with these findings, our previous study [5] has demonstrated that TERT-related anti-apoptotic function was significantly down-regulated in rats with ACR-induced neurobehavioral deficits. The mRNA and protein expression of TERT in the rat cerebral cortex was reduced by ACR treatment [5]. As the critical events in chemical-induced neurodegeneration, oxidative stress and enhanced lipid peroxidation are induced by exposure to ACR, which are also important mechanisms underlying ACR-induced neurotoxicity [11, 12]. During ACR metabolism in the body, excessive levels of reactive oxygen species (ROS) are certainly produced. Moreover, ACR may have deleterious effects on antioxidant enzymes such as superoxide dismutase
(SOD) and glutathione peroxidase (GSH-Px) thus decrease the antioxidant defence in the brains [11, 12]. Furthermore, many evidences $[12,13]$ have shown the production of inflammatory cytokines such as tumor necrosis factor- $\alpha$ (TNF- $\alpha)$ and interleukin-1 $\beta$ (IL-1 $\beta$ ) was enhanced after ACR intoxication.

Accordingly, in recent years, some agents with antiapoptosis, antioxidant and anti-inflammatory properties have been expected to attenuate ACR-induced neurotoxicity $[3,8,11-14]$. As the most active constituent in turmeric, a common spice, with a strong safety record, curcumin has been considered to be a potential natural neuroprotective agent under limelight [15-18]. Based on its known antioxidant, anti-inflammatory and antiapoptosis activities, curcumin has been shown to protect the neurons against cerebral ischemia-reperfusion injury $[15,16]$, dysfunction linked with Parkinson's disease mediated by Bisphenol-A [19], sleep-deprivation induced memory impairments [20], and depression [21], etc. However, there is limited evidence in the possible ameliorative effect of curcumin against ACR-induced neurotoxicity. Prasad and Muralidhara [22] have demonstrated the neuroprotective effect of curcumin in an ACR model of neurotoxicity in an insect species, Drosophila melanogaster. A recently published study [23] reported that curcumin would exert a protective effect against ACR-induced spatial memory impairment in rats. However, the anti-apoptotic, antioxidant and antiinflammatory activities of curcumin have not been well evaluated in ACR-induced neurotoxicity. In the present study, we identified whether curcumin could exert protective effects against neuron apoptosis, oxidative stress and inflammatory response caused by ACR exposure in rats.

\section{Methods \\ Chemicals \\ ACR and curcumin were purchased from Amresco Co. (Solon, OH, USA) and Sigma chemicals Co.(St. Louis, MO, USA), respectively.}

\section{Experimental design}

Male Sprague-Dawley rats, weighing 200-220 g, were obtained from Hubei Experimental Animal Research Center (Hubei, China). Rats were housed in standard translucent cages (5 animals/cage) under controlled 
standard conditions $\left(23 \pm 2{ }^{\circ} \mathrm{C}, 55 \pm 5 \%\right.$ relative humidity, $12 \mathrm{~h}$ light/dark cycle) with restricted access to standard rat chow and free access to tap water. After acclimation for 1-week, healthy animals were randomly assigned into 4 groups (10 rats per group): normal control group; ACR-intoxicated control group; low-dose $(50 \mathrm{mg} / \mathrm{kg})$ curcumin treatment group and high-dose $(100 \mathrm{mg} / \mathrm{kg})$ curcumin treatment group. A dose of $40 \mathrm{mg} / \mathrm{kg}$ ACR (dissolved in normal saline) was intraperitoneally injected every other day for 4 weeks in all animals except the normal control group. The normal rats received saline as control. Meanwhile, rats in the curcumin treatment groups were daily administered with curcumin at the corresponding oral administration dose for 4 weeks. The doses of ACR and curcumin were chosen based on the previous study [5] and preliminary experiments. The normal and ACR-intoxicated control animals were orally administered with the same volume of distilled water. Body weight and behavioral alterations were monitored once a week. At $24 \mathrm{~h}$ after the last administration, all animals were euthanized by $\mathrm{CO}_{2}$ asphyxiation, brain tissues were quickly collected.

\section{Behavioral tests}

All rats were subjected to behavioral analysis to assess their motor functions.

In the hind limb splay examination $[3,5]$, the hind paws of rats were inked, then the rats were placed in a horizontal position of $30 \mathrm{~cm}$ high and dropped onto a white paper. The distance between the center points of right and left heels were recorded as the landing foot spread distance.

In the movement initiation test $[5,24]$, the rat was held by its hind limbs and its torso, one forelimb was lifted above a table in order that the body weight was supported by the other forelimb alone. Then, rat was allowed to initiate stepping movements for one forelimb, and then the other. The averaged time period to initiate one step was recorded as the response latency for each forelimb.

In the gait score test $[3,5]$, animals were placed on the table and were observed for $3 \mathrm{~min}$. Gait was scored as follow: 1: normal gait; 2, slightly abnormal gait characterized by slight ataxia, weakness and foot splay; 3 , moderately abnormal gait characterized by obvious ataxia and foot splay with limb spread during ambulation; 4, severely abnormal gait characterized by a combination of all the above symptoms, dragging hind limbs and inability to support body weight.

\section{Histopathological analysis}

The collected brain tissues were fixed with 10\% neutralbuffered formalin followed by dehydrating and paraffinembedding. Then, embedded brain sections $(5-\mu \mathrm{m}$ thickness) were stained with hematoxylin and eosin (HE) for histopathological observation. The histopathological changes in cerebral cortex, hippocampal CA1, CA3, and dentate gyrus regions were analyzed.

\section{TUNEL assay}

The apoptotic neurons in the brain sections were detected using the terminal deoxynucleotidyl transferase mediated dUTP nick end labelling (TUNEL) assay. After deparaffinization and rehydration, the brain sections were permeabilized with proteinase $\mathrm{K}$ solution, then exposed to the mixture of biotinylated nucleotide dUTP and recombinant terminal deoxynucleotidyl transferase (TdT) following the instruction manual of TUNEL Apoptosis Assay Kit (Servicebio, Wuhan, China). Staining with 4,6-diamino-2-phenyl indole (DAPI) (Sigma, St. Louis, USA) was performed to visualize nuclei. Images were obtained under a fluorescent microscope (Olympus, Center Valley, USA).

\section{Real-time PCR}

Total RNA of brain cerebral cortex tissues was isolated using TRIzol reagent (Invitrogen, Carlsbad, CA, USA). The expression levels of TERT mRNA were measured by real-time PCR using all-in-OneTM qPCR master mix AOPR-1200 (GeneCopoeia, Rockville, MD, USA). The sequences of primer sets for TERT were $5^{\prime}$-TGTTCC TGTTCTGGCTAATGG- 3 '(forward) and 5' -CCTCTT GTGACAGTTCCCGT-3' (reverse). $\beta$-actin gene was applied as a reference.

\section{Immunohistochemistry}

Paraffin-embedded brain sections of $5-\mu \mathrm{m}$ thickness were incubated with a rabbit anti-TERT antibody (Servicebio, Wuhan, China), then a biotinylated goat anti-rabbit secondary antibody (Servicebio, Wuhan, China). Immune complexes were visualized by incubation with 3,3'-diaminobenzidine tetrachloride (DAB) and hematoxylin.

\section{Measurement of parameters related to oxidative stress in cerebral homogenates}

The brain tissue were homogenized with 9 times the volume of PBS on ice and then centrifuged to prepare homogenates. The contents of malondialdehyde (MDA) and glutathione (GSH) as well as the activities of SOD and GSH-Px in the cerebral homogenates were measured following the respective manufacturer's protocols (Nanjing Jiancheng Bio-Engineering Co., Ltd., Nanjing, China). Protein contents in the cerebral homogenates were determined using the bicinchoninic acid assay kit (Nanjing Jiancheng BioEngineering Co., Ltd., Nanjing, China). 


\section{Measurement of IL-1 $\beta$ and TNF- $\alpha$ levels in cerebral homogenates}

The concentrations of IL-1 $\beta$ and TNF-a in cerebral homogenates were determined using using ELISA kits according to the manufacturer's instructions (IL-1 $\beta$ : PeproTech Inc., NJ, USA; TNF-a: R\&D Systems, Minneapolis, MN, USA).

\section{Statistical analysis}

All experiments were conducted with two technical replicates. Data were expressed as the mean $\pm \mathrm{SD}$, and analyzed using one-way analysis of variance (ANOVA) with post hoc Tukey test by SPSS 22.0 software. $P<0.05$ or $P<0.01$ was considered statistically significant.

\section{Results}

\section{Effect of curcumin on ACR-induced body weight and} neurobehavioral changes

As shown in Fig. 1a, the animals in the ACR group began to show slow growth compared to the normal control group since 2 weeks of exposure $(P<0.05)$. At the end of the 4-week exposure period, the average body weight of ACR intoxicated rats was $73.4 \%$ of that of normal rats $(P<0.01)$. However, curcumin administration protected the rats from ACR-induced weigh loss. Compared with the ACR model group, curcumin at the dose of $50 \mathrm{mg} / \mathrm{kg}$ caused a significant weight gain at 4th week $(P<0.05)$. And the body weight of rats administrated with $100 \mathrm{mg} / \mathrm{kg}$ curcumin increased by 12.5 and $14.6 \%$ at 3 rd and 4th week, respectively $(P<0.01)$.

Landing foot spread distance was enlarged rapidly from the first week of ACR exposure (Fig. 1b), and significant differences were found between the ACR intoxicated group and the normal control group throughout the exposure period $(P<0.01)$. Similarly, ACR intoxicated rats developed a progressive impairment of forelimb movement initiation $(P<0.01)$ (Fig. 1c) and significant gait abnormalities (Fig. 1d and e) including obvious ataxia and foot splay, twisting of hind limbs and inability to support body weight. Curcumin intervention in ACR intoxicated rats markedly improved these neurobehavioral changes in a dosedependent manner $(P<0.05 ; P<0.01)$.

\section{Effect of curcumin on ACR-induced histopathological alterations in rat brains}

The neuronal morphological characteristic in the cerebral cortex and hippocampus was identified using H\&E staining. As showing in Fig. 2, severe neuronal loss, condensed and fragmented nuclei were found in the cortex and hippocampus of ACR intoxicated rats. Compared with the ACR model group, there was more nerve cells and less pathological alterations in the brain of rats administrated with curcumin.

\section{Protective effect of curcumin on ACR-induced neuron apoptosis}

As showing in Fig. 3, immunofluorescent staining showed that the number of TUNEL-positive apoptotic nerve cells was significantly increased in the cortex and hippocampus of ACR intoxicated rats. However, curcumin administration could effectively reduce the number of apoptotic cells $(P<0.05 ; P<0.01)$, suggesting its antiapoptotic activity in ACR-damaged neurons. TUNELpositive cells in curcumin-treated ACR intoxicated brains had decreased to approximately $13.8-22.1 \%$ of those in the ACR model group.

\section{Effect of curcumin on ACR-inhibited TERT expression}

Our previous study [5] suggested that TERT, an emerging anti-apoptotic molecule mainly expressed in cortical neurons, was down-regulated in the cerebral cortex of ACR treated rats. In order to identify whether curcumin has regulative effect on ACR-inhibited TERT expression, the mRNA and protein expressions of TERT were detected using real-time PCR and immunohistochemistry, respectively. As shown in Fig. 4, curcumin treatment especially at the dose of $100 \mathrm{mg} / \mathrm{kg}$ increased TERT mRNA expression level $(P<0.01)$, and enhanced the number of TERT-positive cells in ACRintoxicated cortex tissues, suggesting curcumin might exert anti-apoptotic activity in ACR-induced neurotoxicity partly through maintaining TERT-related anti-apoptotic function.

\section{Effect of curcumin on oxidative stress caused by ACR}

To explored the possible anti-oxidant effect of curcumin on ACR-induced neurotoxicity in rats, the contents of MDA, GSH and the activities of SOD, GSH-Px in the cerebral homogenates were quantified as measures of the level of oxidative stress in the brain. As shown in Table 1, the content of MDA was markedly increased, while the GSH level, the activities of SOD and GSHPX were markedly decreased in cerebral homogenates of ACR-treated rats in comparison to the normal control group $(P<0.01)$, suggesting ACR-induced oxidative stress in the brain. As expected, these alterations induced by ACR were significantly ameliorated by curcumin treatment in a dose-dependent manner $(P<0.05 ; P<0.01)$, suggesting that the anti-oxidative activity of curcumin might, at least partly, be responsible for its neuroprotective effect in ACR intoxicated rats.

Effect of curcumin on cerebral contents of IL-1 $\beta$ and TNF$a$ in ACR intoxicated rats

To explore the possible anti-inflammatory activity involved in curcumin mediated neuroprotection in ACR intoxicated rats, the levels of pro-inflammatory 


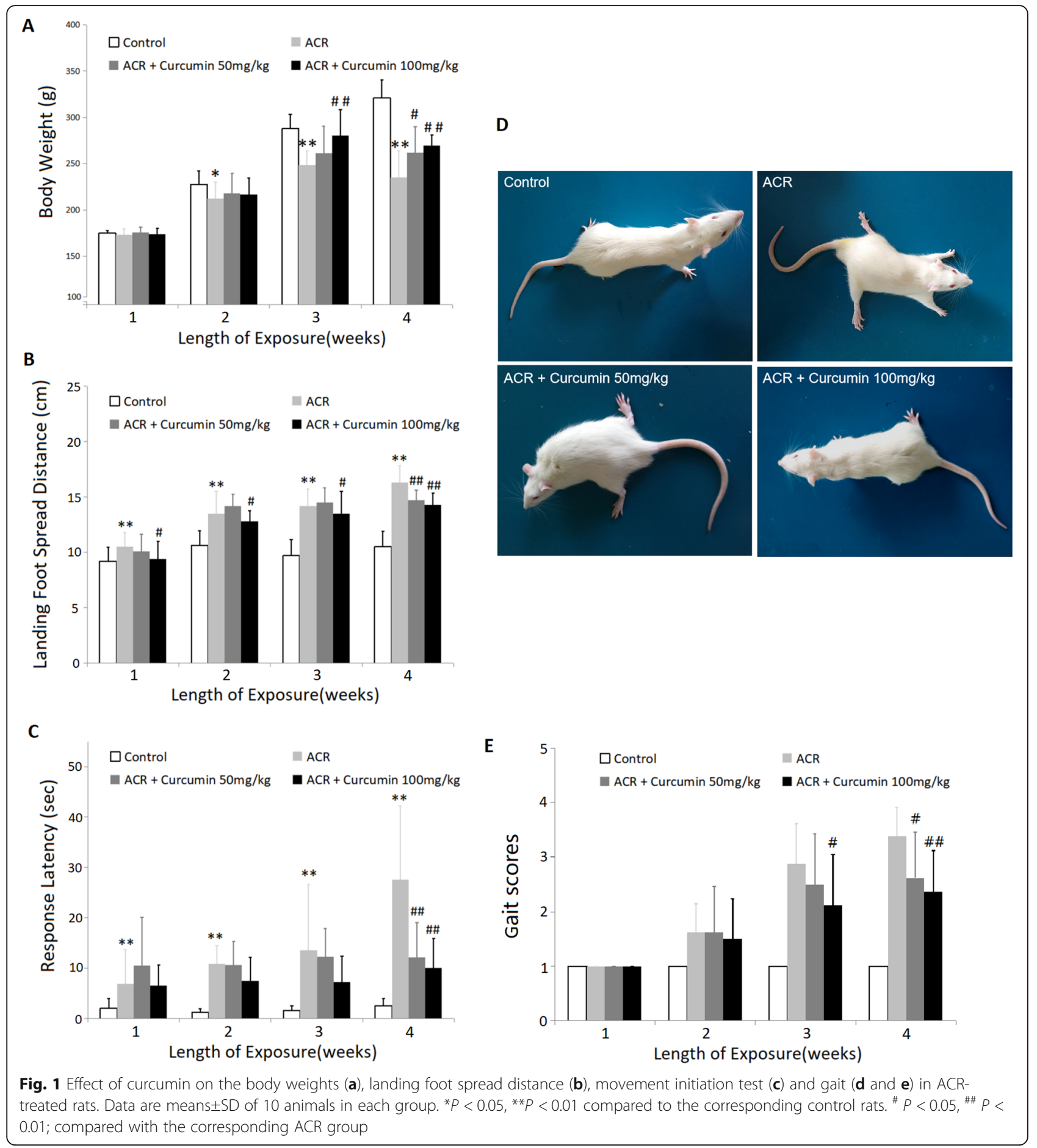

cytokines IL-1 $\beta$ and TNF- $a$ were detected in the cerebral homogenates. Our results show that, although ACR exposure moderately stimulated the production of pro-inflammatory cytokines in brain $(P<0.05)$, curcumin at the dose of $100 \mathrm{mg} / \mathrm{kg}$ significantly decreased the levels of IL-1 $\beta$ and TNF-a by 22.8 and $14.1 \%$, respectively $(P<0.05)$ (Fig. 5$)$, when compared with the ACR group.

\section{Discussion}

Curcumin, with its neuroprotective effects and hardly existing toxicity, have become an attractive alternative treatment tool for various neurological disorders [15-20]. After systemic administration, curcumin can across the blood-brain barrier, and exert its therapeutic efficacy in the brain [25]. In the present study, we demonstrated the anti-apoptotic, antioxidant and anti-inflammatory effects 


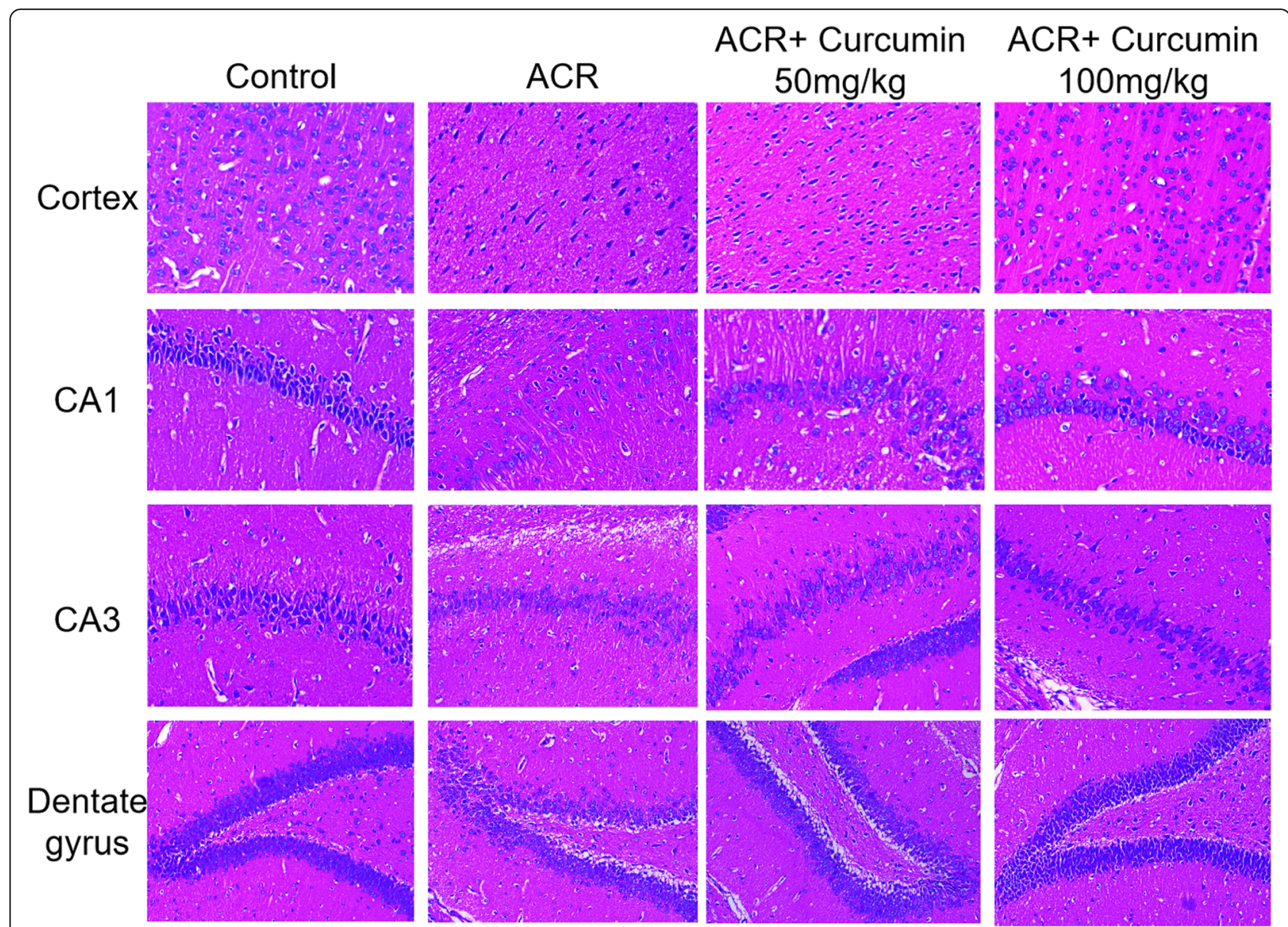

Fig. 2 Effect of curcumin on the histopathological changes in cortex, CA1, CA3, dentate gyrus of ACR-treated rat brains. (H\&E staining 200X)

of curcumin on ACR-induced neurotoxicity in rats, suggesting the use of curcumin to prevent or delay neurological damages induced by ACR exposure. In line with the evidences from humans and animals $[4,5,8,11-14]$, our study showed that the 4-week exposure of rats to ACR at the dose of $40 \mathrm{mg} / \mathrm{kg}$ caused a significant body weight loss, progressive deficits in motor function and adverse pathological outcome in the cortex and hippocampus of rats. Importantly, the present data revealed that curcumin administration could efficiently rescue ACRinduced weight loss and neurobehavioral deficits, relieve the neuropathological damages in brain.

As an important event of neuronal cell number control, apoptosis that is an inappropriate activation of the neuronal cell-suicide program has been well-accepted as a fundamental component in the development of various brain diseases [26]. In particular, in view of the very limited regenerative capacity of the central nervous system tissue, it is vitally important to prevent against neuronal cell apoptosis, and then limit the brain damage caused by neuronal death [26]. So far, apoptosis has become a prime therapeutic target in the development of neuroprotective agents. Treatment preventing the neuronal cell apoptosis can maintain the cell numbers, reduce the severity and progression of brain diseases. In the present study, the anti-apoptotic potential of curcumin in ACR-intoxicated brains which was manifested by the significant decreased TUNEL-positive apoptotic nerve cells in the cortex and hippocampus might be an important mechanism underlying its neuroprotective effect against exposure to ACR.

A variety of small molecules can act on crucial checkpoints of apoptosis [26]. In recent years, the role of TERT in apoptosis has attracted considerable interest as an emerging anti-apoptotic molecule involved in compensatory neuroprotective mechanism against neuronal cell death $[9,10]$. ACR intoxication significantly reduced the expression of TERT in the brain, suggesting the TERTrelated anti-apoptotic function participated in the ACR neurotoxicity [5]. Interestingly, some new evidences showing that curcumin up-regulates function of TERT have emerged [27, 28]. Curcumin extracted with ethyl acetate concentration-dependently up-regulated the TERT mRNA expression in rat clone-9 hepatocytes [27]. 

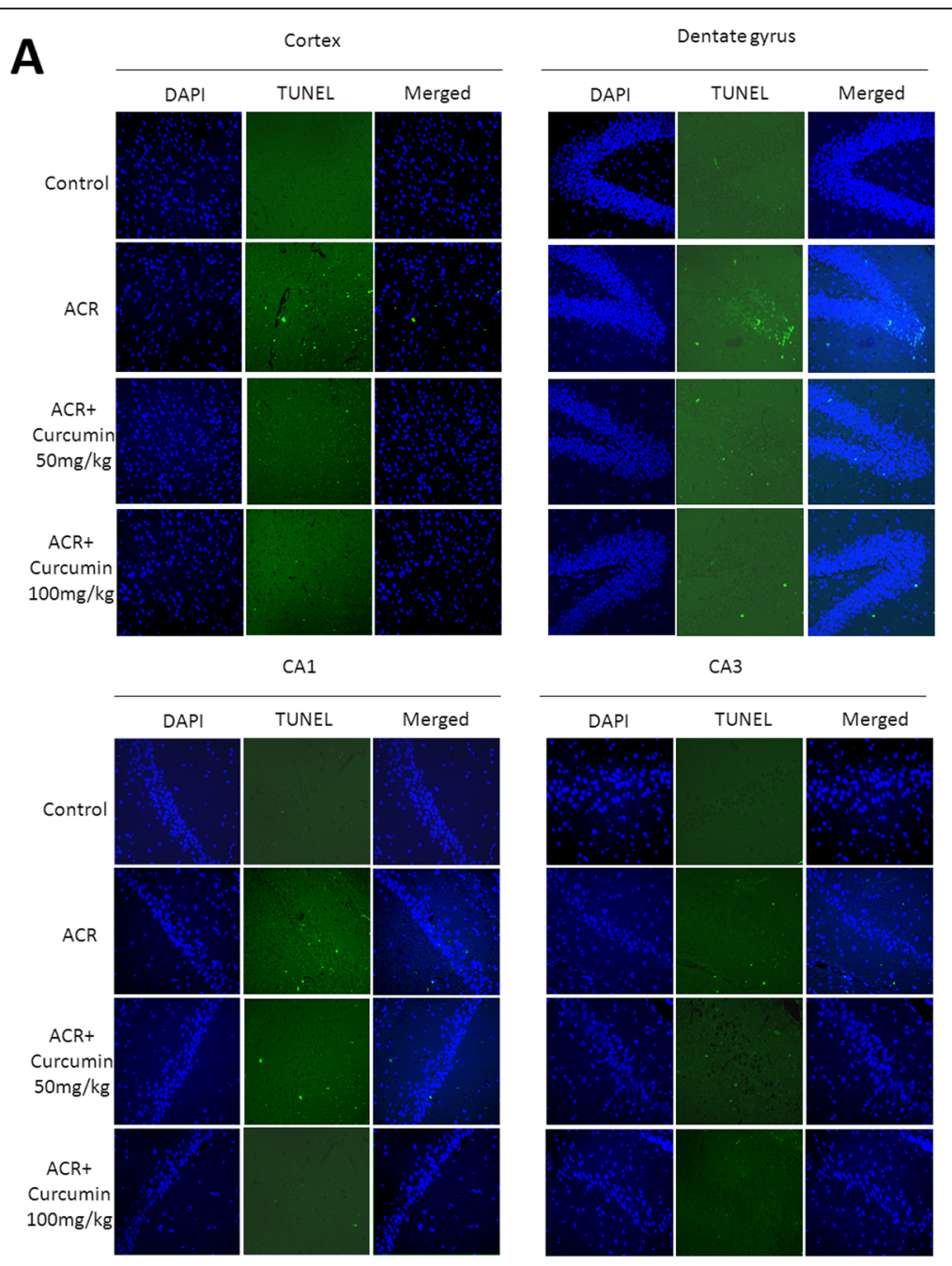

B

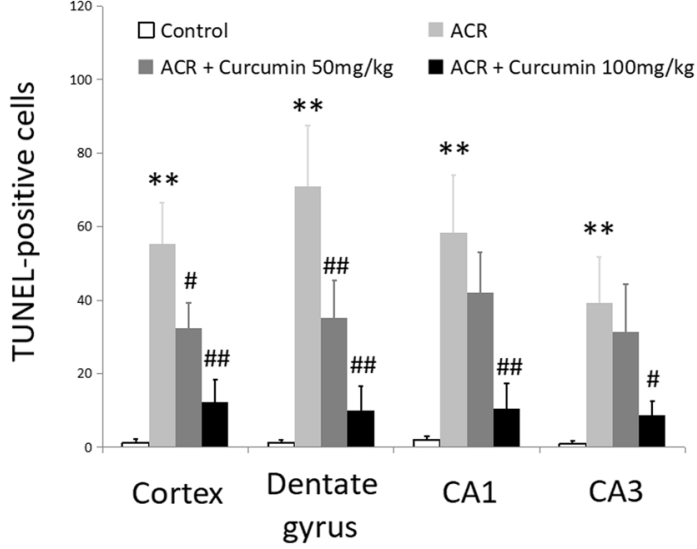

Fig. 3 Effect of curcumin on the neuron apoptosis in ACR-treated rat brains. (TUNEL staining 400x). a Representative images. b Quantitative assessment of neuronal density of TUNEL-positive cells (number of cells $/ \mathrm{mm}^{2}$ ). Data are means \pm SD of 10 animals in each group. ${ }^{* *} P<0.01$ compared to the corresponding control rats. ${ }^{\#} P<0.05$, \#\# $P<0.01$; compared with the corresponding ACR group

Pirmoradi et al. [28] reported that the TERT expression of rat adipose tissue-derived stem cells was significantly increased in the presence of curcumin at concentrations of 1 and $5 \mu \mathrm{M}$. In line with these in vitro studies [27, 28], we showed the curcumin-induced in vivo up-regulation of TERT at the levels of gene and protein, which might be one mechanism underlying the anti-apoptotic activity of curcumin in ACR-intoxicated brains. 
A

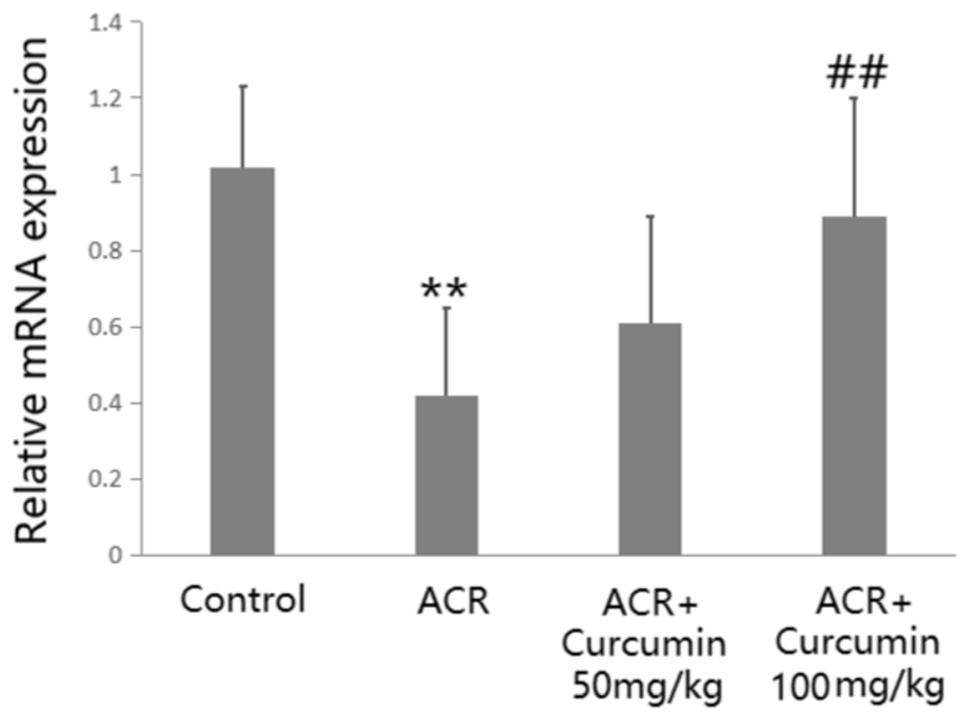

B
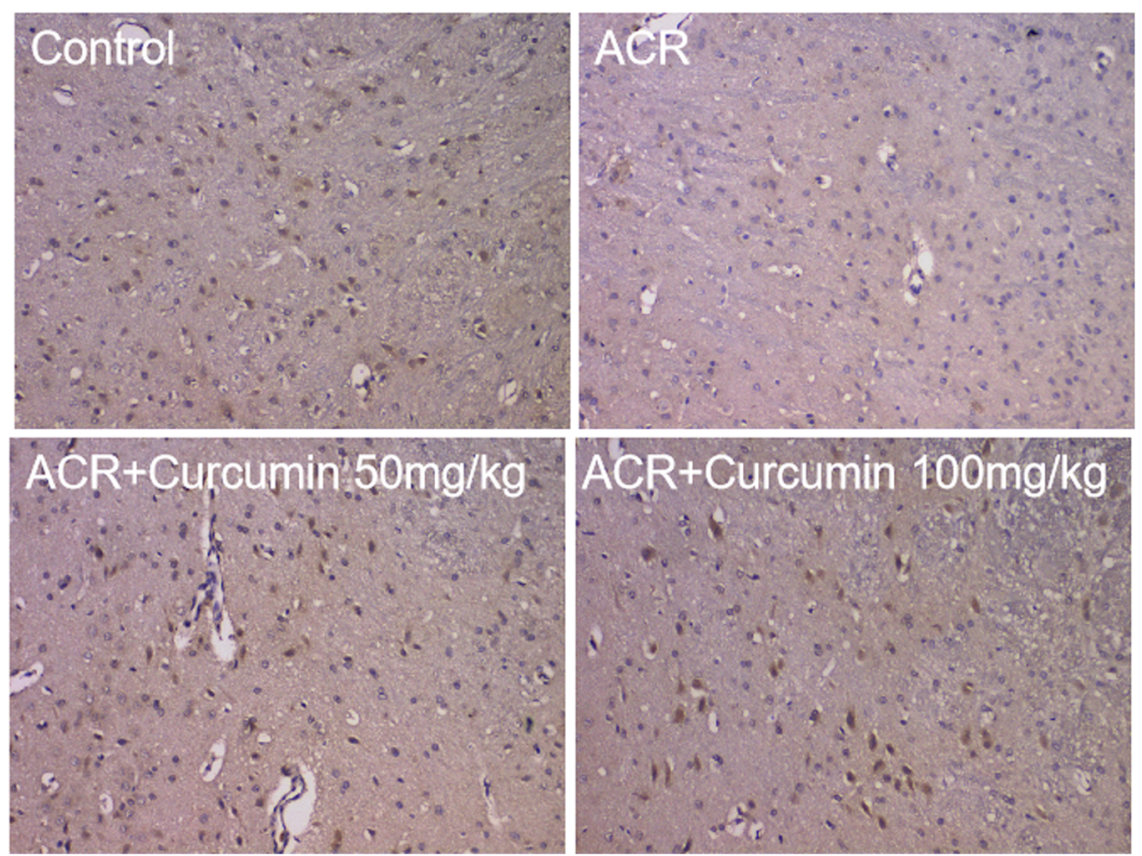

Fig. 4 Effect of curcumin on the expression of TERT in the cortex tissues of ACR-treated rats. a The mRNA expression was measured with Realtime PCR. $\mathbf{b}$ Immunohistochemical staining for the protein expression of TERT. Data are means \pm SD of 10 animals in each group. ${ }^{* *} P<0.01$ compared to the corresponding control rats. ${ }^{\#} P<0.01$; compared with the corresponding ACR group

Table 1 Effect of curcumin on the levels of MDA, GSH, SOD and GSH-Px in cerebral homogenates prepared from ACR intoxicated rats $(n=10$, mean \pm SD)

\begin{tabular}{|c|c|c|c|c|}
\hline Groups & $\begin{array}{l}\text { MDA } \\
\text { (nmol/mg prot) }\end{array}$ & $\begin{array}{l}\text { GSH } \\
\text { (mg/g prot) }\end{array}$ & $\begin{array}{l}\text { SOD } \\
\text { (U/mg prot) }\end{array}$ & $\begin{array}{l}\text { GSH-Px } \\
\text { (U/mg prot) }\end{array}$ \\
\hline Normal & $0.425 \pm 0.141$ & $4.41 \pm 0.58$ & $60.21 \pm 5.38$ & $14.81 \pm 1.95$ \\
\hline$A C R$ & $1.133 \pm 0.352 * *$ & $2.30 \pm 0.47^{* *}$ & $52.72 \pm 6.94 * *$ & $10.36 \pm 1.84 * *$ \\
\hline $\mathrm{ACR}+$ curcumin $50 \mathrm{mg} / \mathrm{kg}$ & $0.918 \pm 0.322$ & $2.77 \pm 0.46^{\#}$ & $53.89 \pm 8.02$ & $12.58 \pm 1.96^{\#}$ \\
\hline $\mathrm{ACR}+$ curcumin $100 \mathrm{mg} / \mathrm{kg}$ & $0.854 \pm 0.216^{\#}$ & $2.92 \pm 0.59^{\#}$ & $59.16 \pm 6.46^{\#}$ & $13.15 \pm 1.87$ \#\# \\
\hline
\end{tabular}

${ }^{* *} P<0.01$ compared to the corresponding control rats. ${ }^{\#} P<0.05,{ }^{\# \#} P<0.01$; compared with the corresponding ACR group 
IL-1 $\beta$

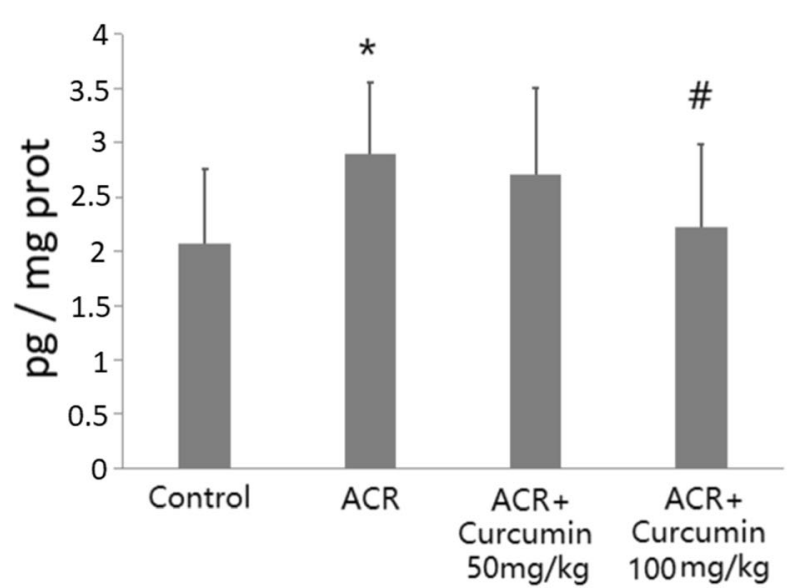

TNF-a

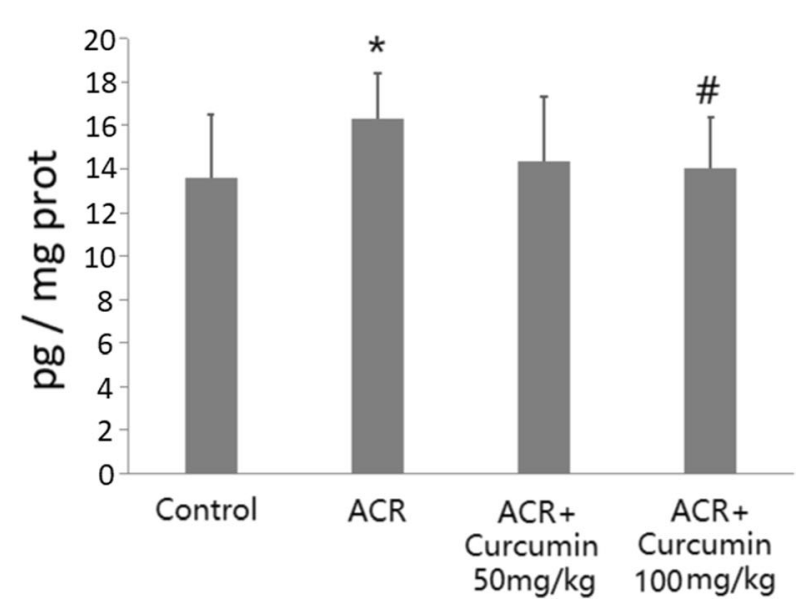

Fig. 5 Effect of curcumin on cerebral contents of IL-1 $\beta$ and TNF-a in ACR intoxicated rats. Data are means \pm SD of 10 animals in each group. ${ }^{*} P<0.05$ compared to the corresponding control rats. $\# P<0.05$; compared with the corresponding ACR group

In addition, curcumin is well known for its classic and strong anti-oxidative and anti-inflammatory activities [29]. ACR exposure has been demonstrated to result in a disturbance in the balance between the free radical formation and elimination, the latter of which is mediated by antioxidant systems $[11,12]$. The phenolic structure in curcumin confers electron-capturing properties, which destabilize ROS, explaining the well-accepted antioxidant effects [30]. However, being similar to other antioxidants including vitamin $\mathrm{E}$, vitamin $\mathrm{C}$, and carotenoids, curcumin has been found to show double-edged roles in the level of intracellular ROS, which appeared to be highly dependent on the cell type [30-32]. Curcumin has been reported to elevate ROS levels in multiple cancer cells [30-32]. In this study, in line with the wellaccepted anti-oxidative activity of curcumin in normal and non-malignant cells [29-32], 4-week exposure of rats to $40 \mathrm{mg} / \mathrm{kg}$ ACR markedly enhanced the level of MDA (an essential biomarker of oxidative stress and lipid peroxidation), decreased the content of GSH (a biologically important intracellular thiol acting as a free radical scavenger) and the activities of SOD and GSH-Px (two important antioxidant enzymes) in the brain tissues. But curcumin alleviated the augmented production of MDA and the reduction of antioxidant capacity induced by ACR, thus might play a role in the detoxification of reactive oxygen species generated by ACR. Moreover, neuroinflammation has been demonstrated in various pathologies of the brain including ACR-induced neurotoxicity [33]. The 4-week exposure to ACR induced inflammatory responses in the brain tissues, evident by upregulated levels of IL- $1 \beta$ and TNF-a, two potent pro-inflammatory cytokines acting as master regulators of neuroinfammation in the central nerve system.
While curcumin could improve the ACR-induced neuroinflammation, which was in accord with its proven antiinflammatory property.

\section{Conclusions}

In summary, this study convinced the anti-apoptotic, antioxidant and anti-inflammatory effects of curcumin on ACR-induced neurotoxicity in rats. And maintaining TERT-related anti-apoptotic function might be one mechanism underlying the protective effect of curcumin on ACR-intoxicated brains.

\section{Abbreviations}

ACR: Acrylamide; GSH: Glutathione; GSH-Px: Glutathione peroxidase; HE: Hematoxylin and eosin; IL-1 $\beta$ : Interleukin-1 $\beta$; MDA: Malondialdehyde; ROS: Reactive oxygen species; SOD: Superoxide dismutase; TERT: Telomerase reverse transcriptase; TNF-a: Tumor necrosis factor-a; TUNEL: Terminal deoxynucleotidyl transferase mediated dUTP nick end labelling

\section{Acknowledgements \\ Not applicable.}

\section{Authors' contributions}

JW and XC contributed to the design of the research. JG,XH and SL performed the research. JG, CX and JW analyzed the data. JW prepared the article. All authors read and approved the final manuscript.

\section{Funding}

This study was financially supported by the National Natural Science Funding of China (Nos. 71974153, Nos. 81602108). The study funder had no further role in the study design, data collection, analyses, interpretation of results, writing of the article, or the decision to submit it for publication.

\section{Availability of data and materials}

The datasets supporting the conclusions of this article are included within the article. The raw data can be requested from the corresponding author. 
Technology (resolution number 2019078), and accomplished in line with the guidelines of the National Health and Medical Research Council of China.

\section{Consent for publication}

Not applicable.

\section{Competing interests}

The authors declare that they have no competing interests.

Received: 21 October 2019 Accepted: 11 August 2020

Published online: 18 August 2020

\section{References}

1. Erkekoglu P, Baydar T. Acrylamide neurotoxicity. Nutr Neurosci. 2014;17(2): 49-57.

2. Koszucka A, Nowak A, Nowak I, Motyl I. Acrylamide in human diet, its metabolism, toxicity, inactivation and the associated European Union legal regulations in food industry. Crit Rev Food Sci Nutr. 2019;25:1-16.

3. Su B, Guan Q, Wang M, Liu N, Wei X, Wang S, Yang X, Jiang W, Xu M, Yu S. Calpeptin is neuroprotective against acrylamide-induced neuropathy in rats. Toxicology. 2018;400-401:1-8.

4. Pennisi M, Malaguarnera G, Puglisi V, Vinciguerra L, Vacante $M$, Malaguarnera M. Neurotoxicity of acrylamide in exposed workers. Int J Environ Res Public Health. 2013:10(9):3843-54

5. Wang J, Zhang MY, Xu SQ, Cheng J, Yu ZJ, Hu XM. Down-regulation of telomerase reverse transcriptase-related anti-apoptotic function in a rat model of acrylamide induced neurobehavioral deficits. Biotech Histochem. 2018;93(7):512-8.

6. Sahinturk V, Kacar S, Vejselova D, Kutlu HM. Acrylamide exerts its cytotoxicity in NIH/3T3 fibroblast cells by apoptosis. Toxicol Ind Health. 2018;34(7):481-9.

7. Lee JG, Wang YS, Chou CC. Acrylamide-induced apoptosis in rat primary astrocytes and human astrocytoma cell lines. Toxicol in Vitro. 2014;28(4): $562-70$

8. Sun G, Wang X, Li T, Qu S, Sun J. Taurine attenuates acrylamide- induced apoptosis via a PI3K/AKT-dependent manner. Hum Exp Toxicol. 2018;37(12): 960327118765335

9. Liu MY, Nemes A, Zhou QG. The emerging roles for telomerase in the central nervous system. Front Mol Neurosci. 2018;11:160.

10. Li J, Tang B, Qu Y, Mu D. Telomerase reverse transcriptase: a novel neuroprotective mechanism involved in neonatal hypoxic-ischemic brain injury. Int J Dev Neurosci. 2011;29(8):867-72.

11. Goudarzi M, Mombeini MA, Fatemi I, Aminzadeh A, Kalantari H, Nesari A, Najafzadehvarzi H, Mehrzadi S. Neuroprotective effects of Ellagic acid against acrylamide-induced neurotoxicity in rats. Neurol Res. 2019;41(5):41928.

12. Santhanasabapathy R, Vasudevan S, Anupriya K, Pabitha R, Sudhandiran G. Farnesol quells oxidative stress, reactive gliosis and inflammation during acrylamide-induced neurotoxicity: behavioral and biochemical evidence. Neuroscience. 2015;308:212-27.

13. Acaroz U, Ince S, Arslan-Acaroz D, Gurler Z, Kucukkurt I, Demirel HH, Arslan $\mathrm{HO}$, Varol N, Zhu K. The ameliorative effects of boron against acrylamideinduced oxidative stress, inflammatory response, and metabolic changes in rats. Food Chem Toxicol. 2018;118:745-52.

14. Adewale OO, Brimson JM, Odunola OA, Gbadegesin MA, Owumi SE, Isidoro C, Tencomnao T. The potential for plant derivatives against acrylamide neurotoxicity. Phytother Res. 2015;29(7):978-85.

15. Mukherjee A, Sarkar S, Jana S, Swarnakar S, Das N. Neuro-protective role of nanocapsulated curcumin against cerebral ischemia-reperfusion induced oxidative injury. Brain Res. 1704;2019:164-73.

16. Xu L, Ding L, Su Y, Shao R, Liu J, Huang Y. Neuroprotective effects of curcumin against rats with focal cerebral ischemia-reperfusion injury. Int J Mol Med. 2019:43(4):1879-87.

17. Baj T, Seth R. Role of Curcumin in regulation of TNF-a mediated brain inflammatory responses. Recent Patents Inflamm Allergy Drug Discov. 2018; 12(1):69-77.

18. Zhao XC, Zhang L, Yu HX, Sun Z, Lin XF, Tan C, Lu RR. Curcumin protects mouse neuroblastoma Neuro-2A cells against hydrogen-peroxide-induced oxidative stress. Food Chem. 2011;129(2):387-94.

19. Akintunde JK, Farouk AA, Mogbojuri O. Metabolic treatment of syndrome linked with Parkinson's disease and hypothalamus pituitary gonadal hormones by turmeric curcumin in Bisphenol-a induced neuro-testicular dysfunction of wistar rat. Biochem Biophys Rep. 2018;17:97-107.

20. Noorafshan A, Karimi F, Kamali AM, Karbalay-Doust S, Nami M. Restorative effects of curcumin on sleep-deprivation induced memory impairments and structural changes of the hippocampus in a rat model. Life Sci. 2017; 189:63-70.

21. Fan C, Song Q, Wang P, Li Y, Yang M, Yu SY. Neuroprotective effects of Curcumin on IL-1 1 -induced neuronal apoptosis and depression-like behaviors caused by chronic stress in rats. Front Cell Neurosci. 2019;12:516.

22. Prasad SN. Muralidhara. Neuroprotective effect of geraniol and curcumin in an acrylamide model of neurotoxicity in Drosophila melanogaster: relevance to neuropathy. J Insect Physiol. 2014;60:7-16.

23. Yan D, Yao J, Liu Y, Zhang X, Wang Y, Chen X, Liu L, Shi N, Yan H. Tau hyperphosphorylation and P-CREB reduction are involved in acrylamideinduced spatial memory impairment: suppression by curcumin. Brain Behav Immun. 2018;71:66-80.

24. Pang Y, Lin S, Wright C, Shen J, Carter K, Bhatt A, Fan LW. Intranasal insulin protects against substantia nigra dopaminergic neuronal loss and alleviatesmotor deficits induced by 6-OHDA in rats. Neuroscience. 2016;318: $157-65$.

25. Goozee KG, Shah TM, Sohrabi HR, Rainey-Smith SR, Brown B, Verdile G, Martins RN. Examining the potential clinical value of curcumin in the prevention and diagnosis of Alzheimer's disease. Br J Nutr. 2016;115(3):44965.

26. Cavallucci V, D'Amelio M. Matter of life and death: the pharmacological approaches targeting apoptosis in brain diseases. Curr Pharm Des. 2011; 17(3):215-29.

27. Pan MH, Wu JC, Ho CT, Badmaev V. Effects of water extract of Curcuma longa (L.) roots on immunity and telomerase function. J Complement Integr Med. 2017;14(3):20150107.

28. Pirmoradi S, Fathi E, Farahzadi R, Pilehvar-Soltanahmadi Y, Zarghami N. Curcumin affects adipose tissue-derived Mesenchymal stem cell aging through TERT gene expression. Drug Res (Stuttg). 2018;68(4):213-21.

29. He Y, Yue $Y$, Zheng $X$, Zhang $K$, Chen $S$, Du Z. Curcumin, inflammation, and chronic diseases: how are they linked? Molecules. 2015;20(5):9183-213.

30. Willenbacher E, Khan SZ, Mujica SCA, Trapani D, Hussain S, Wolf D, Willenbacher W, Spizzo G, Seeber A. Curcumin: new insights into an ancient ingredient against Cancer. Int J Mol Sci. 2019;20(8):1808.

31. Tong R, Wu X, Liu Y, Liu Y, Zhou J, Jiang X, Zhang L, He X, Ma L. Curcumininduced DNA Demethylation in human gastric Cancer cells is mediated by the DNA-damage response pathway. Oxidative Med Cell Longev. 2020;2020: 2543504.

32. Liczbiński P, Michałowicz J, Bukowska B. Molecular mechanism of curcumin action in signaling pathways: Review of the latest research. Phytother Res. 2020. https://doi.org/10.1002/ptr.6663.

33. Zong C, Hasegawa R, Urushitani M, Zhang L, Nagashima D, Sakurai T, Ichihara S, Ohsako S, Ichihara G. Role of microglial activation and neuroinflammation in neurotoxicity of acrylamide in vivo and in vitro. Arch Toxicol. 2019;93(7):2007-19.

\section{Publisher's Note}

Springer Nature remains neutral with regard to jurisdictional claims in published maps and institutional affiliations.

Ready to submit your research? Choose BMC and benefit from:

- fast, convenient online submission

- thorough peer review by experienced researchers in your field

- rapid publication on acceptance

- support for research data, including large and complex data types

- gold Open Access which fosters wider collaboration and increased citations

- maximum visibility for your research: over $100 \mathrm{M}$ website views per year

At $\mathrm{BMC}$, research is always in progress.

Learn more biomedcentral.com/submissions 